\title{
Extra-anatomic bypass of the superior vena cava after successful stenting for fibrosing mediastinitis
}

\author{
Eugenio Pompeo, MD, Franco Stella, MD, Arnaldo Ippoliti, MD, and Tommaso C. Mineo, MD, Rome, Italy
}

Supplemental material is available online.

$\mathrm{F}$ ibrosing mediastinitis is a rare benign disorder with an etiology that commonly remains unknown, although tuberculosis, syphilis, histoplasmosis, and blastomycosis have been all considered potential etiologic factors. It is characterized by focal or diffuse proliferation of acellular collagen and fibrous tissue within the mediastinum, which mimic malignancy. About $40 \%$ of patients have the disease discovered incidentally during their young-middle age, but the rest have symptoms of compression of mediastinal structures, the superior vena cava being the most common clinically. ${ }^{1}$

Until a few years ago, superior vena cava replacement was the only effective treatment for superior vena cava syndrome resulting from fibrosing mediastinitis.

From the Division of Thoracic Surgery, Policlinico Tor Vergata University, Rome, Italy.

Received for publication July 16, 2007; accepted for publication Aug 7 , 2007.

Address for reprints: Eugenio Pompeo, MD, Cattedra di Chirurgia Toracica, Policlinico Università Tor Vergata, V.le Oxford, 81, 00133 Rome, Italy (E-mail: pompeo@med.uniroma2.it).

J Thorac Cardiovasc Surg 2008;135:220-1

$0022-5223 / \$ 34.00$

Copyright $\odot 2008$ by The American Association for Thoracic Surgery doi:10.1016/j.jtcvs.2007.08.051
More recently, endovascular angioplasty and stenting has offered a new, attractive, and patient-friendly therapeutic option, although questions remain regarding long-term outcome and the optimal treatment strategy for stenting failures. ${ }^{2,3}$ In fact, removal of the stent is often impossible in these instances and superior vena cava resection and reconstruction can result in a technically demanding surgical procedure, particularly when the fibrotic mass involves other vital mediastinal structures such as pulmonary artery and main stem bronchi.

Herein we report the case histories of 2 patients with idiopathic fibrosing mediastinitis who underwent initial successful superior vena cava stenting but had subsequent occlusion of the stent, which was treated by a nonresectional extra-anatomic bypass of the superior vena cava.

\section{Clinical Summaries}

PAtient 1. A 45-year-old woman was admitted with dyspnea and dilated veins over the chest.

The patient history began 6 years earlier when she had superior vena cava syndrome. A computed tomographic (CT) scan of the chest showed a mediastinal mass involving the middle and anterosuperior mediastinum encasing and compressing the superior vena cava and the left innominate vein. Surgical biopsy through anterior mediastinotomy led to diagnosis of fibrosing mediastinitis. At that time, the patient was treated by endovascular placement of a metal stent in the superior vena cava, which resulted in disappearance of symptoms. The patient remained free of symptoms for 3 years, but afterward neck swelling, dyspnea, and chest vein dilatation recurred. A chest CT scan demonstrated a slight enlargement of the mediastinal mass with occlusion of the stent and development of superior-inferior vena cava collateral circulation (Figure 1).

After admission to our department, it was decided to perform an extra-anatomic bypass of the superior vena cava, which was easily carried out without resection of the mediastinal mass (Figure 2, $A$ ). Total operative time was 90 minutes and the patient was
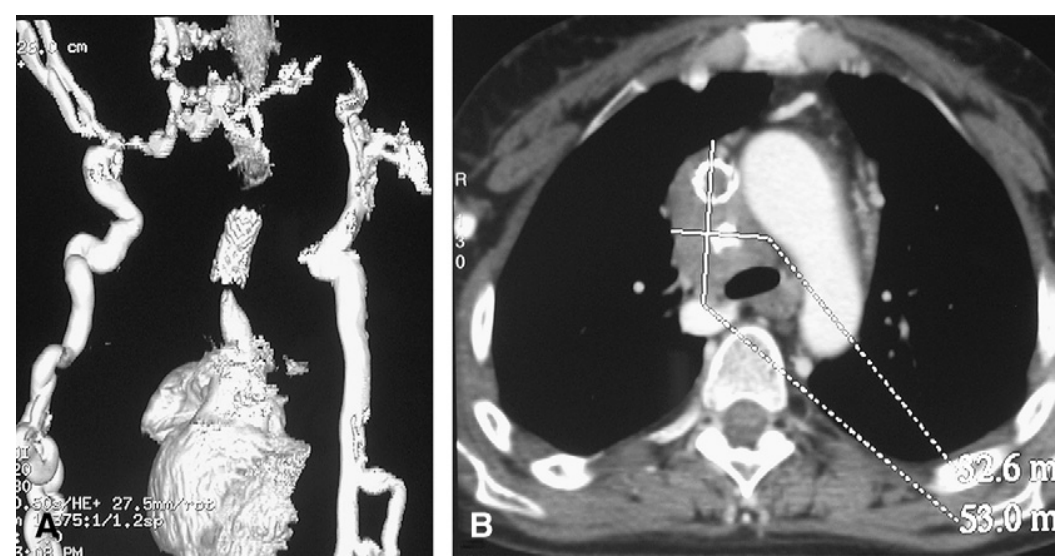

Figure 1. Preoperative computed tomographic scan: $A$, An angiogram shows absence of flow in the stented superior vena cava with development of superior-inferior vena cava collateral circulation via right lateral thoracic and hemiazygous veins. B, Axial view illustrates the mediastinal fibrotic mass encasing the occluded metal stent. 

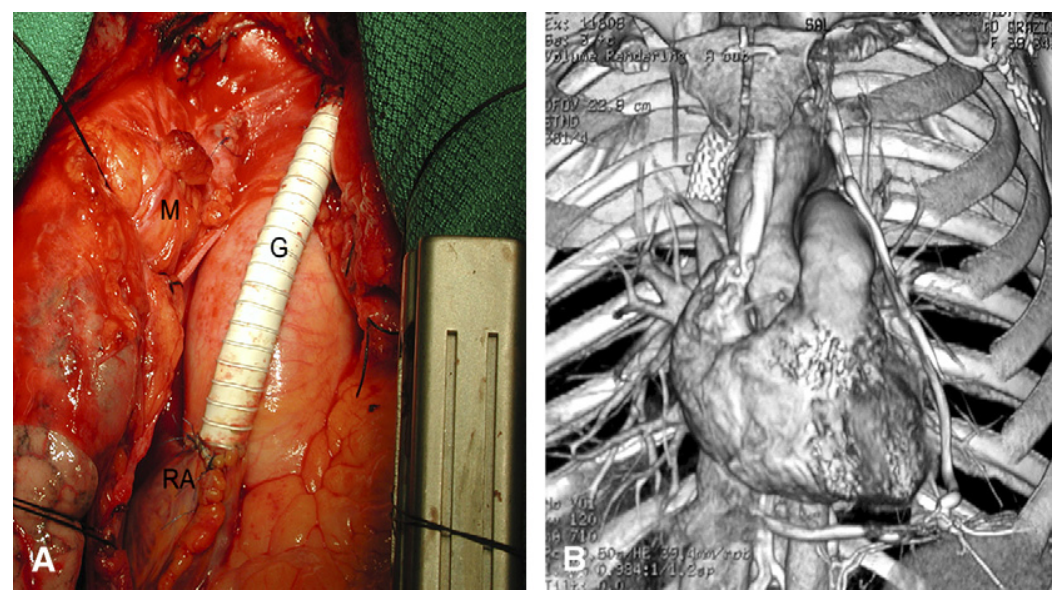

Figure 2. A, Intraoperative view of the extra-anatomic polytetrafluoroethylene graft bypass $(G)$, right atrium $(R A)$, and fibrotic mass encasing the stented superior vena cava (M). B, Coronal 3-dimensional reconstruction of the mediastinum depicts both the occluded metal stent and patent polytetrafluoroethylene graft.

discharged 4 days after the operation on a regimen of anticoagulant therapy.

A radiogram obtained 36 months after the operation showed a fully patent graft (Figure 2, $B$ ) and a relatively stable mediastinal mass (Figure E1).

PATIENT 2. A 48-year-old woman had a cough and swollen, dilated veins of the face and upper extremities. She had normal lung function and blood tests. A CT scan confirmed a mass in the mediastinum and videomediastinoscopy permitted diagnosis of fibrosing mediastinitis. Endovascular stenting of the superior vena cava resulted in resolution of symptoms. Eighteen months later symptoms recurred owing to obstruction of the stent, and extra-anatomic superior vena cava bypass was successfully carried out as previously described. So far, the patient remains free of symptoms with a patent graft 14 months after the operation.

\section{Discussion}

In patients with superior vena cava obstruction owing to fibrosing mediastinitis, either vena cava replacement or endovascular stenting has been used with satisfactory results and primary patency rates of $63 \%{ }^{2}$ and $21 \%,^{3}$ respectively.

We have hypothesized that a step-by-step strategy including initial stenting followed by subsequent vena cava replacement at the reappearance of disabling symptoms could assure more prolonged benefits than each single modality per se. It is also conceivable that a multistep approach including initial stenting, subsequent bypass, and additional secondary endovascular treatment might be considered as well.

We also believed that whenever radiologic imaging shows a focal disease, an extra-anatomic bypass performed with no resection of the vena cava, and possibly distant from the mediastinal lesion, could shorten the operative time and minimize the risk of an early involvement of the graft because of disease progression.

Efficacy of polytetrafluoroethylene for superior vena cava replacement in benign disorders has been questioned, assuming better results with venous autografts. ${ }^{4}$ However, within our surgical experience with superior vena cava replacement, ${ }^{5}$ we have used polytetrafluoroethylene since 1987 with satisfactory results. In these instances, to minimize the risk of graft occlusion, we tailor anticoagulants to keep the international normalized ratio between 2.8 and 3.0.

In conclusion, an integrated treatment strategy entailing initial stenting followed by nonresectional extra-anatomic bypass of the superior vena cava allowed prolonged benefit in 2 patients with severely symptomatic fibrosing mediastinitis.

\section{References}

1. Rossi SE, Mc Adams HP, Rosado-deChristenson ML, Franks TJ, Galvin JR, et al. Fibrosing mediastinitis. Radiographics. 2001;21:737-57.

2. Kalra M, Gloviczki P, Andrews JC, Cherry KJ Jr, Bower TC, Panneton JM, et al. Open and endovascular treatment of superior vena cava syndrome caused by nonmalignant disease. J Vasc Surg. 2003;38: 215-23.

3. Bakken AM, Protack CD, Saad WE. Long-term outcomes of primary angioplasty and primary stenting of central venous stenosis in hemodialysis patients. J Vasc Surg. 2007;45:776-83.

4. Doty JR, Flores JH, Doty DB. Superior vena cava obstruction: bypass using spiral vein graft. Ann Thorac Surg. 1999;67:1111-6.

5. Ricci C, Benedetti-Valentini F, Fiorani $\mathrm{P}$, et al. La chirurgia riparativa della vena cava superiore. Gazz Intern Med Chir. 1970;21:1551-75. 

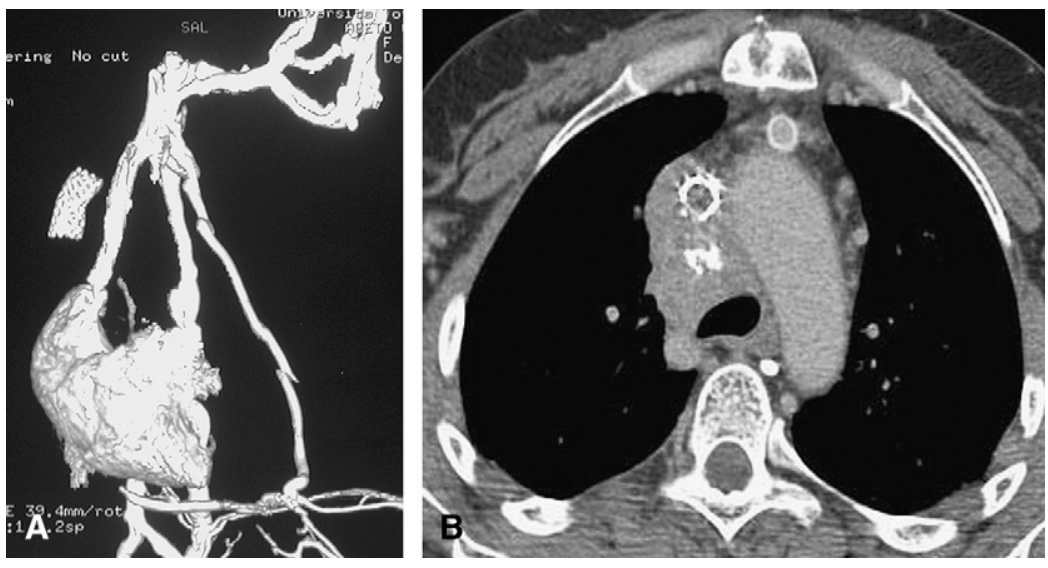

Figure E1. Postoperative computed tomographic scan at $\mathbf{3 6}$ months: A, An angiogram shows the patent graft with normalization of the collateral circulation depicted preoperatively. B, Axial view illustrates the relatively stable mediastinal mass and the graft, which lies in the anterior mediastinum but distant from the disease process. 